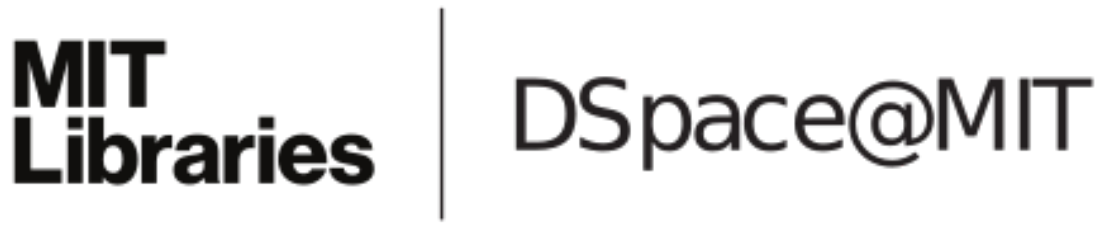

\author{
MIT Open Access Articles
}

Deformation Response of Catenated DNA

Networks in a Planar Elongational Field

The MIT Faculty has made this article openly available. Please share how this access benefits you. Your story matters.

As Published: 10.1021/ACSMACROLETT.0C00360

Publisher: American Chemical Society (ACS)

Persistent URL: https://hdl.handle.net/1721.1/135319

Version: Author's final manuscript: final author's manuscript post peer review, without publisher's formatting or copy editing

Terms of use: Creative Commons Attribution-Noncommercial-Share Alike 


\title{
Deformation Response of Catenated DNA Networks in a Planar Elongational Field
}

\author{
Beatrice W. Soh and Patrick S. Doyle*
}

Cite This: ACS Macro Lett. 2020, 9, 944-949

Read Online

\begin{abstract}
ACCESS
Wll Metrics \& More

Article Recommendations

ABSTRACT: A kinetoplast is a complex catenated DNA network that bears resemblance to a two-dimensional polymeric system. In this work, we use single-molecule experiments to study the transient and steady-state deformation of kinetoplasts in a planar elongational field. We demonstrate that kinetoplasts deform in a stagewise manner and undergo transient deformation at large strains, due to conformational rearrangements from an intermediate metastable state. Kinetoplasts in an elongational field achieve a steady-state deformation that depends on strain rate, akin to the deformation of linear polymers. We do not observe an abrupt transition between the nondeformed and deformed states of a kinetoplast, in contrast to the coil-stretch transition for a linear polymer.
\end{abstract}

Supporting Information

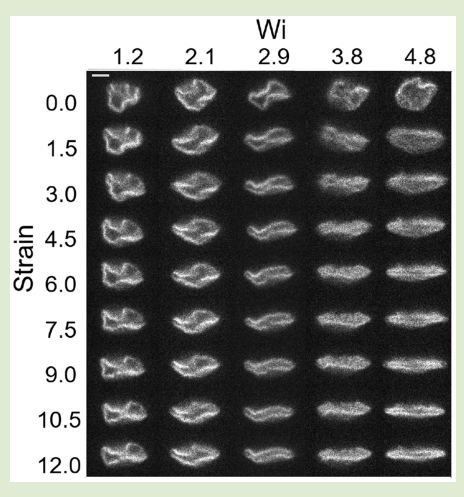

A fundamental aspect of polymer physics is to understand the effects of molecular topology on the dynamics of polymeric materials. It is well-known that the rheological response of entangled linear polymers ${ }^{1}$ is drastically different from that of entangled ring ${ }^{2}$ and branched polymers. ${ }^{3,4}$ To date, most studies on polymer dynamics have focused on linear, ring, and branched polymers. The dynamics of entangled polymers, such as knotted $^{5-7}$ and topologically linked ring polymers, ${ }^{8,9}$ has also garnered much attention. With the emerging interest in two-dimensional materials for their unique physical and chemical properties, ${ }^{10}$ coupled with recent advances in synthesizing planar macromolecules, ${ }^{11}$ there is a desire to study polymers with planar structures, for example, polycatenanes $^{12,13}$ and Olympic gels. ${ }^{14}$

Single-molecule techniques provide a powerful approach for studying the link between polymer topology and dynamics. ${ }^{15}$ Over the past few decades, single-molecule experiments have been widely used to probe the behavior of double-stranded DNA as a model polymer. Through the direct visualization and precise manipulation of individual DNA molecules, singlemolecule experiments have been instrumental in addressing key concepts of polymer physics that are not easily investigated using bulk ensemble methods, such as molecular individualism $^{16,17}$ and conformational hysteresis. ${ }^{18}$ Single-molecule studies of polymers with different topologies are enabled by the physical and biological characteristics of DNA, which allow molecular biology tools to be leveraged for the preparation of DNA with nonlinear topologies, in particular, ring ${ }^{19}$ and branched DNA. ${ }^{20}$ While polymers with nonlinear topologies have been investigated on the single-molecule level, there are yet to be studies on polymers with complex, sheet-like structures, primarily due to the lack of a suitable experimental system.

Recently, our group proposed the kinetoplast as a model system for studying two-dimensional polymer systems. ${ }^{21} \mathrm{~A}$ kinetoplast is a complex network of topologically interlinked circular DNA, consisting of a few thousand minicircles (0.5$2.9 \mathrm{kbp})$ and several dozen maxicircles $(20-40 \mathrm{kbp}){ }^{22} \mathrm{~A}$ kinetoplast from the trypanosomatid Crithidia fasciculata has approximately 5000 minicircles $(\sim 2.5 \mathrm{kbp})$ and 25 maxicircles $(\sim 40 \mathrm{kbp}) .^{23}$ Each minicircle is linked on average to three other minicircles (i.e., minicircle valence of three), and the topologically linked maxicircles are threaded through the minicircles, hence, forming a network within a network. ${ }^{22}$ When visualized by fluorescence microscopy, kinetoplasts in good solvents appear as cup-shaped sheets with a diameter of $\sim 5 \mu \mathrm{m}$ and thickness of $\sim 3 \mu \mathrm{m}$. ${ }^{21}$ Found in the mitochondrial DNA of trypanosomatid parasites, the kinetoplast has attracted considerable interest from a microbiology point of view, ${ }^{22-25}$ but little is known about the material properties of the network structure.

A two-dimensional polymer is a topologically planar sheet with laterally connected repeat units. ${ }^{10}$ A kinetoplast is a twodimensional catenated network that bears resemblance to a two-dimensional polymeric system. One minicircle has a fully

Received: May 11, 2020

Accepted: June 10, 2020 
stretched contour length of $<5$ Kuhn lengths and can be viewed as an effective bond in a coarse-grained model of a twodimensional polymer. Our previous work showed that kinetoplasts deform elastically when squeezed into a microfluidic channel with weakly confining dimensions. ${ }^{21}$ In this study, we use single-molecule experiments to investigate the deformation response of kinetoplasts in a planar elongational field. We observe the transient and steady-state deformation dynamics of kinetoplasts subjected to a planar elongational field and use the well-studied dynamics of linear polymers as a framework for understanding the deformation of kinetoplasts. Our results demonstrate that, like linear polymers, kinetoplasts in a planar elongational field achieve a steady-state deformation that is dependent on strain rate. Unlike linear polymers, kinetoplasts can undergo transient conformational changes at large accumulated strains, owing to the interplay between topology, bending resistance, thermal fluctuations, and force imposed by the field gradient.

In this study, experiments were conducted in $5 \mu \mathrm{m}$ tall crossslot polydimethylsiloxane (PDMS) channels. Independently applied potentials to opposite arms of the cross-slot channel allowed for control over the location of the stagnation point and manual trapping of kinetoplasts at the stagnation point for long observation times. ${ }^{26-28}$ Kinetoplast DNA from Crithidia fasciculata (TopoGEN) was stained with fluorescent dye YOYO-1 at a base pair to dye ratio of $8: 1$ and diluted into experimental buffer containing 4 vol $\% \beta$-mercaptoethanol and $0.1 \% 10 \mathrm{kDa}$ polyvinylpyrrolidone in $0.5 \times$ Tris-boric acidEDTA solution. Molecules were visualized using an inverted Zeiss Axiovert microscope with a $63 \times 1.4$ NA oil-immersed objective, and images were acquired by a Photometrics Prime 95B sCMOS camera. See Supporting Information (SI) for additional experimental details.

According to the principle of electrohydrodynamic equivalence proposed by Long et al., ${ }^{29}$ the electrophoretic stretching force on a DNA molecule is equivalent to the hydrodynamic drag force exerted by a flow, with the hydrodynamic velocity in the flow field equal to the electrophoretic velocity in the electric field. The kinematics of a planar elongational electric field is hence described by ${ }^{26-28}$

$$
\begin{aligned}
& v_{x}=\mu E_{x}=\dot{\epsilon} x \\
& v_{y}=\mu E_{y}=-\dot{\epsilon} y
\end{aligned}
$$

where $v_{x}$ and $v_{y}$ are the velocities in the $x$ and $y$ directions, respectively, $\mu$ is the electrophoretic mobility, $E_{x}$ and $E_{y}$ are the electric fields in the $x$ and $y$ directions, respectively, and $\dot{\epsilon}$ is the strain rate. The imposed strain rate was calibrated against applied voltage $e^{26}$ and changed by varying the applied voltage. The deformation of a polymer molecule in an elongational field can be characterized by the Weissenberg number (Wi), which describes the ratio of elastic to viscous forces and is defined as the product of strain rate and longest relaxation time of the polymer molecule, $\tau$ :

$$
\mathrm{Wi}=\dot{\epsilon} \tau
$$

The replication of a kinetoplast involves the replication of each minicircle and maxicircle in the network, followed by the segregation into two equal-sized progeny networks. ${ }^{23}$ During replication of the minicircles, the size of the network does not change, which is hypothesized to result in the observed increase in network density and increase in minicircle valence from three to six. After replication of the minicircles is complete, the network goes through a remodeling process, during which the network doubles in size and the minicircle valence decreases to three. The replicated network then splits to form two daughter networks. ${ }^{22,24} \mathrm{~A}$ population of kinetoplasts consists of kinetoplasts isolated at different stages of the replication cycle, hence, we observe kinetoplasts of a range of sizes, with equilibrium major axis lengths ranging between 4 and $11 \mu \mathrm{m}$. Despite the heterogeneity in underlying structure and connectivity, each kinetoplast represents a catenated DNA network. The overall goal of this work is to gain insight into the deformation of two-dimensional catenated networks by studying the deformation of kinetoplasts.

Each experiment consisted of deforming a kinetoplast at various strain rates and observing the relaxation of the kinetoplast following weak perturbation from equilibrium. A kinetoplast was electrophoretically driven to the stagnation point of the cross-slot channel and allowed to relax at equilibrium for $\sim 30 \mathrm{~s}$. The field was switched on and the kinetoplast was subjected to a strain rate between 0.7 and 1.2 $\mathrm{s}^{-1}$. After observing the kinetoplast for at least $1 \mathrm{~min}$, the field was switched off and the kinetoplast was allowed to relax back to equilibrium. The strain rate was then increased and the procedure was repeated. Kinetoplasts on the small end of the size range do not deform significantly within the experimentally feasible range of strain rates, hence we opted to examine larger kinetoplasts, kinetoplasts with an equilibrium major axis length $\geq 7.5 \mu \mathrm{m}$, for this study (see $\mathrm{SI}$ ).

Inspired by methods to quantify the deformation of a red blood cell, ${ }^{30,31}$ we measure the extent of deformation of a kinetoplast by the deformation index (DI), defined as

$$
\mathrm{DI}=\frac{L_{\mathrm{M}}-L_{\mathrm{m}}}{L_{\mathrm{M}}+L_{\mathrm{m}}}
$$

where $L_{\mathrm{M}}$ and $L_{\mathrm{m}}$ are the lengths of the major and minor axes, respectively. The DI can vary between 0 and 1 , where 0 represents a circle and 1 represents an infinitely thin rod. We highlight that because kinetoplasts are rotationally asymmetric, the measured DI is dependent on the orientation of the kinetoplast with respect to the viewing plane. ${ }^{21}$ The use of 5 $\mu \mathrm{m}$ tall channels in this study served to weakly confine the larger kinetoplasts and generally orient them to be either face up or face down at equilibrium.

To measure the relaxation time of a kinetoplast, the kinetoplast was weakly perturbed from equilibrium at a strain rate between 1.2 and $1.7 \mathrm{~s}^{-1}$, and the major and minor axis lengths of the kinetoplast during relaxation back to equilibrium were observed. Multiple deform-relax cycles were performed for each kinetoplast. The evolution of average DI with time during the relaxation process was fit to a single-exponential decay to extract a relaxation time for each kinetoplast. The measured relaxation times for the ensemble of kinetoplasts studied range from 0.4 to $1.8 \mathrm{~s}$ (see SI for more details).

Due to differences in topology and size, each individual kinetoplast has a different deformation behavior. Figure 1 shows the deformation trajectories of a select kinetoplast at various $\mathrm{Wi}$, based on which we can make two key observations. First, the kinetoplast attains a steady-state deformation at large strain $(\epsilon=\dot{\epsilon} \mathrm{t})$ in an elongational field. As seen from Figure 1a, for all Wi considered, the DI versus strain curves plateau at a steady-state value by 50 units of strain. Second, the larger the $\mathrm{Wi}$, the greater the extent of kinetoplast deformation. This is evidently seen by comparing the snapshots of the kinetoplast at steady-state in an elongational field of $\mathrm{Wi}=1.2$ versus 4.8 . At 


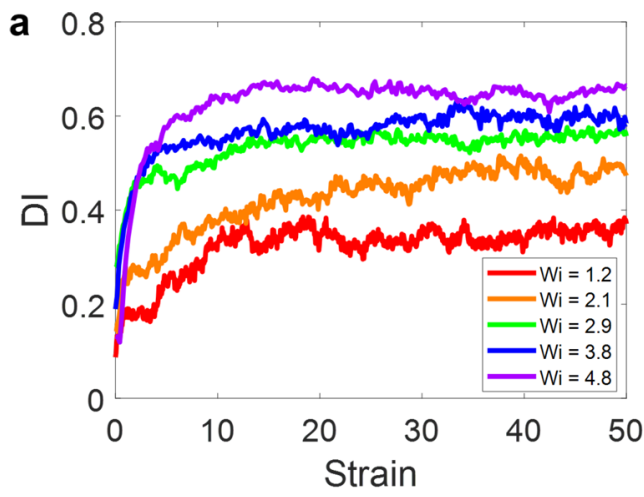

b

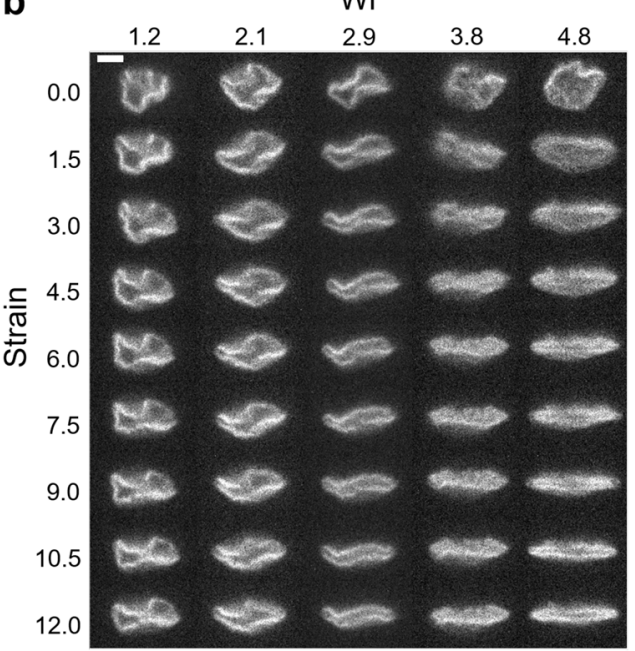

Figure 1. Stretching of a select kinetoplast. (a) Deformation index (DI) vs accumulated strain $(\epsilon=\dot{\epsilon} \mathrm{t})$ at different $\mathrm{Wi}$. The relaxation time of the kinetoplast is $1.7 \mathrm{~s}$. (b) Snapshots of the kinetoplast corresponding to the traces in (a). Scale bar represents $5 \mu \mathrm{m}$.

$\mathrm{Wi}=1.2$, the deformed kinetoplast bears some resemblance to its equilibrium shape, with the two lobes of the structure clearly visible; at $\mathrm{Wi}=4.8$, the kinetoplast takes on a highly elongated shape, with the two lobes being tightly squeezed together such that the kinetoplast appears as a flattened disk. We note that such deformation behavior is generally similar to that observed with linear polymers, ${ }^{16,17}$ as well as ring, ${ }^{32}$ branched, ${ }^{33}$ and knotted polymers. ${ }^{28}$ See Figures S6 and S7 in the SI for additional deformation trajectories.

Next, we consider in detail the transient deformation dynamics of kinetoplasts. As seen from Figure 2, the deformation trajectories of the six kinetoplasts subjected to different Wi share several features. When the planar elongational field is turned on, the kinetoplasts deform sharply to a certain state, typically within two strain units. The kinetoplasts can stay in a given state for tens of strain units before undergoing further deformation. For example, the kinetoplast depicted in Figure $2 c$ experiences about 75 units of strain before going through a second deformation to a more elongated shape. In comparison, linear lambda bacteriophage DNA with a contour length of $22 \mu \mathrm{m}$ typically achieves steadystate extension in a planar elongational field within 10 strain units. ${ }^{16,17,34}$ We note that the deformation of kinetoplasts is elastic and all kinetoplasts eventually relax to equilibrium when the field is turned off.

To probe the stagewise deformation of kinetoplasts that can occur over large accumulated strains, we focus on the transient deformation of a select kinetoplast (Figure 3). We consider the evolution of the major and minor axis lengths with strain, in addition to the DI. Shortly after the field is turned on, the kinetoplast undergoes the first stage of deformation and its major and minor axis lengths quickly attain steady values. After about 40 units of strain, the kinetoplast goes through a folding event in which a rim of the kinetoplast folds over and into its body. The kinetoplast becomes more compact along the compressional axis of the field, resulting in a noticeable decrease in the minor axis length and consequent increase in DI.

In an elongational field, the conformation of a kinetoplast is determined by a competition between the force exerted by the field gradient, entropic elasticity, and bending resistance. When a kinetoplast is subjected to an elongational field, the topological complexity of the catenated network coupled with the forces acting on it can lead to the kinetoplast becoming trapped in a metastable intermediate state. Eventually, thermal fluctuations can allow the force imposed by the field gradient to overcome the bending resistance of the kinetoplast and drive the system to a state of lower free energy, thereby involving rearrangement of the kinetoplast conformation. Figure 3 depicts an example of a kinetoplast that remains in a kinetically trapped state before undergoing a conformational rearrangement of the network structure, which manifests as a folding event. While the elongation and compression of a linear polymer are coupled, a kinetoplast can undergo compression without elongation via rearrangement of the network conformation. In this case, it is the compressional axis of the planar elongational field that drives the folding event and further deformation of the kinetoplast. It is interesting to note that simulations of polyelectrolytes with strong counterion couplings in electric fields have shown that molecules can become trapped in metastable backfolded states during the transition from a compact to an elongated state. ${ }^{35}$ We highlight that it is possible for the kinetoplast to rotate out-of-plane during deformation, but the changes in major and minor axis lengths associated with reorientation are small relative to the dramatic changes induced by conformational rearrangement. See SI for further discussion and movies (movies S1, S2, and S3) of transient kinetoplast deformation.

Having examined the transient deformation of kinetoplasts, we now consider the steady-state dynamics of kinetoplasts in a planar elongational field. All kinetoplasts were observed for at least 50 units of strain at each Wi. If the kinetoplast exhibits stagewise deformation, the steady-state DI is the average DI at the plateau of the final distinct stage. Otherwise, the steadystate DI is taken to be the average DI of the kinetoplast after 20 strain units. We point out that the reported steady-state DI values can represent the kinetoplast in a metastable or stable state. Figure 4 presents a plot of the steady-state DI for an ensemble of 21 kinetoplasts as a function of Wi. Given the heterogeneity in underlying topology of the kinetoplasts, it is unsurprising that the DI-Wi curves of the different kinetoplasts do not collapse onto a universal curve. Nonetheless, we observe overall characteristics from the ensemble DIWi curves that are similar to that for a linear polymer. Notably, the steady-state DI values generally increase with $\mathrm{Wi}$ and plateau at high Wi. The maximum DI, which ranges between 0.32 and 0.64 for the kinetoplasts in this study (Figure 4, inset), is attained at $\mathrm{Wi} \sim 1$.

To further investigate the steady-state deformation dynamics of kinetoplasts, we look at plots of DI versus Wi for select 
a
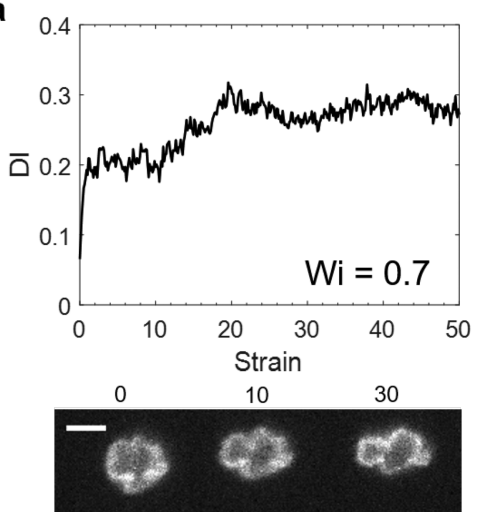

d

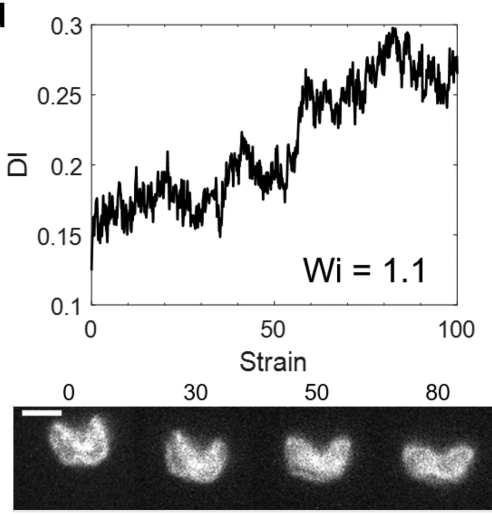

b

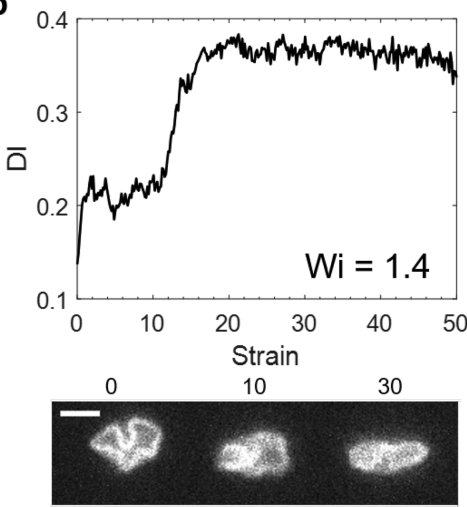

e

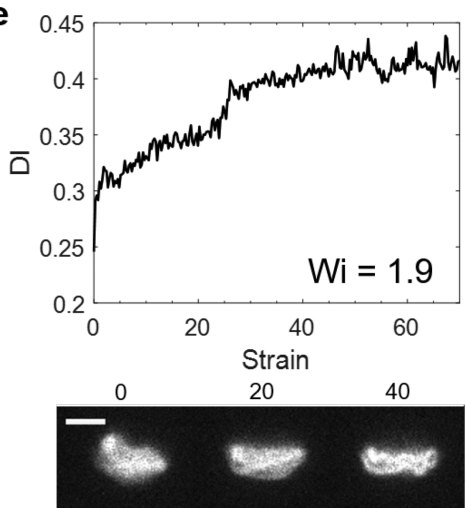

C

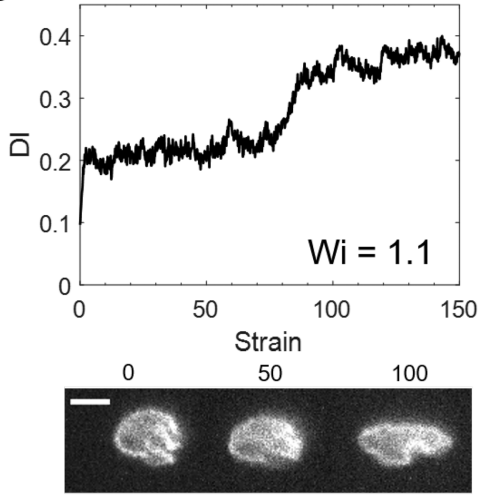

f
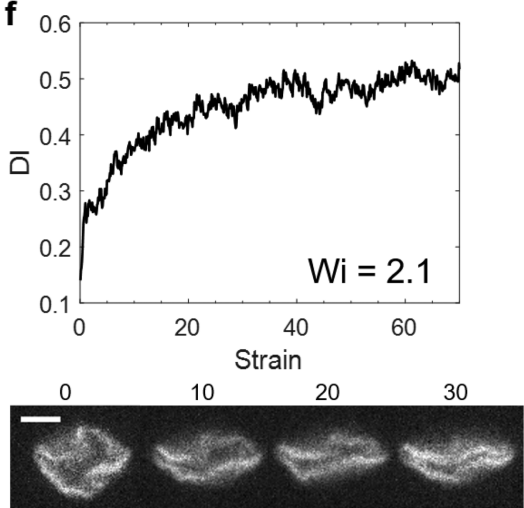

Figure 2. DI vs strain trajectories for six select kinetoplasts and snapshots of the kinetoplasts at various accumulated strains as labeled. Scale bars represent $5 \mu \mathrm{m}$.
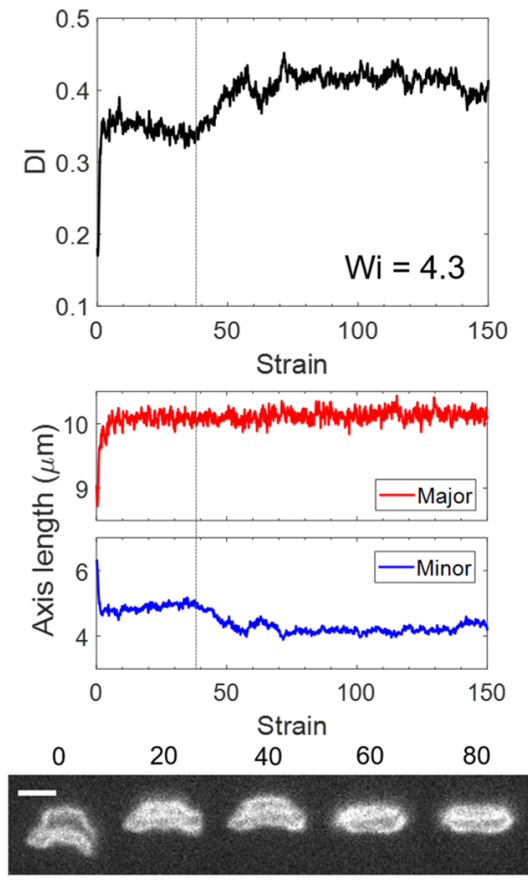

Figure 3. DI, major axis length and minor axis length versus strain trajectories for a select kinetoplast. The vertical dashed line indicates when the kinetoplast undergoes a folding event. Snapshots of the kinetoplast at various accumulated strains as labeled. Scale bar represents $5 \mu \mathrm{m}$.

individual kinetoplasts (Figure 5). Based on the individual DIWi plots, the deformation versus Wi curves for kinetoplasts do not appear to take on a universal shape, with some kinetoplasts

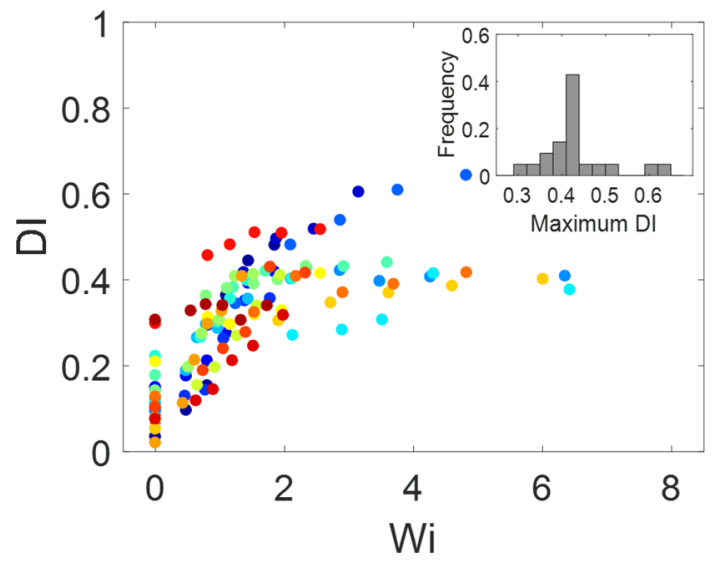

Figure 4. Steady-state DI vs Wi for an ensemble of 21 kinetoplasts. Error bars represent $95 \%$ confidence intervals and are smaller than the symbols. Inset: Histogram of maximum DI attained by the kinetoplasts.

deforming sharply over a narrow Wi range (Figure 5a,b) and others deforming more gradually with increasing Wi (Figure $5 \mathrm{c}-\mathrm{e})$. In a planar elongational field, linear polymers display a sharp coil-stretch transition. ${ }^{16,17}$ A feature of the coil-stretch transition is an increase in steady-state chain extension fluctuations in the vicinity of the critical strain rate, with the peak magnitude of fractional extension fluctuations reaching $\sim 0.1 .^{28,36}$ We can examine the steady-state conformational fluctuations for kinetoplasts by measuring the standard deviation of the steady-state DI values (Figure 5, inset). As seen from the standard deviation of steady-state DI versus Wi plots, there is no discernible trend in conformational 


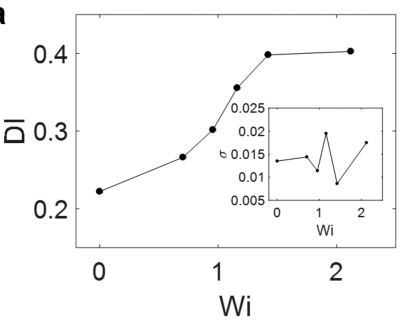

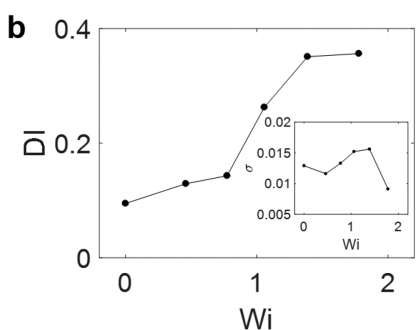

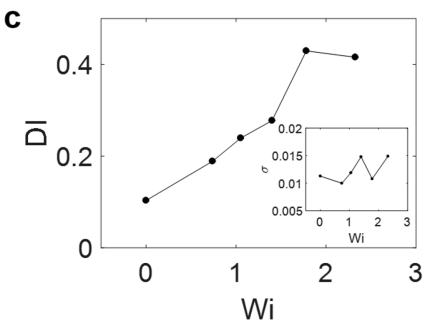

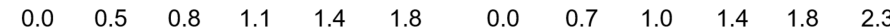

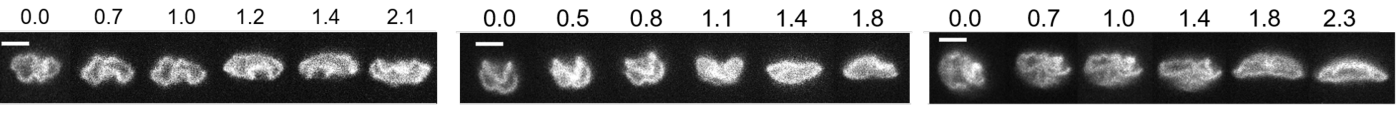

d

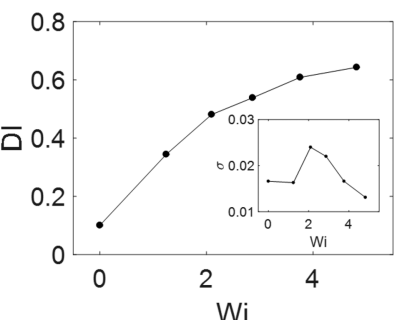

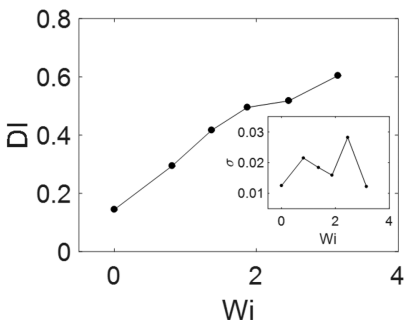
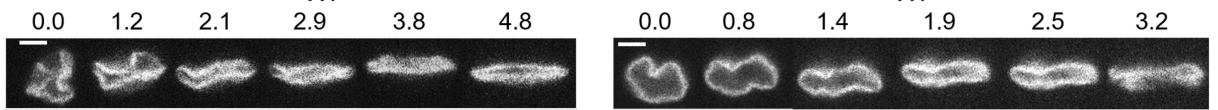

Figure 5. Steady-state DI vs Wi for five select kinetoplasts and snapshots of the kinetoplasts at each specified Wi. The lines are drawn to guide the eye. Scale bars represent $5 \mu \mathrm{m}$. Error bars represent $95 \%$ confidence intervals and are smaller than the symbols. Inset: Standard deviation of steadystate DI $(\sigma)$ vs Wi.

fluctuations with respect to $\mathrm{Wi}$. In particular, for the kinetoplasts in Figure 5a,b that undergo a sharp transition from a nondeformed to deformed state, the degree of conformational fluctuations remains relatively constant over the range of Wi considered.

While analogies between linear polymers and kinetoplasts arise naturally, the physical similarities between kinetoplasts and red blood cells in terms of shape and size also motivate comparisons between their dynamics. Our previous study estimated the bending rigidity of kinetoplasts to be $1.8 \times 10^{-19}$ $\mathrm{J},{ }^{21}$ identical to the membrane bending modulus found for red blood cells. ${ }^{37}$ When subjected to an elongational flow imposed by a hyperbolic contraction in a microchannel, red blood cells elongate from a circular to elliptical shape, with the degree of deformation dependent on the flow rate. The DI values of the red blood cells increase with particle Reynolds number ( $\mathrm{Re}$ ) and begin to plateau at $\operatorname{Re} \sim 1 .^{38}$ The saturated DI value for red blood cells at high elongational stress is reported to be about $0.6,^{30}$ similar to the maximum DI values attained with kinetoplasts. The red blood cells relax back to their original shape after flowing through the hyperbolic contraction, ${ }^{30,38}$ an indication of elastic deformation. It is striking to see both quantitative and qualitative similarities in the deformation behaviors of kinetoplasts and red blood cells, despite the fundamental differences in structure.

In this work, we studied the transient and steady-state deformation dynamics of kinetoplasts in a planar elongational field. Unexpectedly, we demonstrated that kinetoplasts can undergo stagewise deformation at large strains, as a result of conformational rearrangements from a metastable intermediate state. Kinetoplasts can attain a steady-state deformation, and the extent of deformation increases with Wi. By examining the conformational fluctuations of kinetoplasts at steady-state, we note the absence of an abrupt transition between the nondeformed and the deformed states, in contrast to the coil-stretch transition for a linear polymer. Our study provides valuable insight into the deformation behavior of a twodimensional catenated network, and we hope that this motivates future studies into the dynamics of kinetoplasts, which can be used to understand the dynamics of twodimensional polymers.

\section{ASSOCIATED CONTENT}

\section{SI Supporting Information}

The Supporting Information is available free of charge at https://pubs.acs.org/doi/10.1021/acsmacrolett.0c00360.

Experimental, image processing, and data analysis details; Size range of kinetoplasts; Additional deformation trajectories of kinetoplasts; Further discussion of transient deformation and out-of-plane rotation of kinetoplasts (PDF)

Movie S1: Transient deformation of the kinetoplast presented in Figure 2c (MP4)

Movie S2: Transient deformation of the kinetoplast presented in Figure $2 \mathrm{f}$ (MP4)

Movie S3: Transient deformation of the kinetoplast presented in Figure 3 (MP4)

\section{AUTHOR INFORMATION}

\section{Corresponding Author}

Patrick S. Doyle - Department of Chemical Engineering, Massachusetts Institute of Technology, Cambridge, Massachusetts 02139, United States; 이이. orcid.org/0000-00032147-9172; Email: pdoyle@mit.edu

Author

Beatrice W. Soh - Department of Chemical Engineering, Massachusetts Institute of Technology, Cambridge, 
Massachusetts 02139, United States; 이이.org/0000-00018399-5995

Complete contact information is available at: https://pubs.acs.org/10.1021/acsmacrolett.0c00360

\section{Notes}

The authors declare no competing financial interest.

\section{ACKNOWLEDGMENTS}

This work was supported by the National Science Foundation (NSF) Grant CBET-1936696. B.W.S. is funded by the Agency for Science, Technology and Research (A*STAR), Singapore. We acknowledge Alexander Klotz and Ahmed Khorshid for useful discussions.

\section{REFERENCES}

(1) Doi, M.; Edwards, S.F. The Theory of Polymer Dynamics; Clarendon Press: Oxford, 1988.

(2) Kapnistos, M.; Lang, M.; Vlassopoulos, D.; Pyckhout-Hintzen, W.; Richter, D.; Cho, D.; Chang, T.; Rubinstein, M. Unexpected Power-Law Stress Relaxation of Entangled Ring Polymers. Nat. Mater. 2008, 7 (12), 997-1002.

(3) Fetters, L. J.; Kiss, A. D.; Pearson, D. S.; Quack, G. F.; Vitus, F. J. Rheological Behavior of Star-Shaped Polymers. Macromolecules 1993, 26 (4), 647-654.

(4) Daniels, D. R.; McLeish, T. C. B.; Crosby, B. J.; Young, R. N.; Fernyhough, C. M. Molecular Rheology of Comb Polymer Melts. 1. Linear Viscoelastic Response. Macromolecules 2001, 34 (20), 70257033.

(5) Tubiana, L.; Rosa, A.; Fragiacomo, F.; Micheletti, C. Spontaneous Knotting and Unknotting of Flexible Linear Polymers: Equilibrium and Kinetic Aspects. Macromolecules 2013, 46 (9), 36693678

(6) Caraglio, M.; Micheletti, C.; Orlandini, E. Stretching Response of Knotted and Unknotted Polymer Chains. Phys. Rev. Lett. 2015, 115 (18), 188301.

(7) Soh, B. W.; Klotz, A. R.; Robertson-Anderson, R. M.; Doyle, P. S. Long-Lived Self-Entanglements in Ring Polymers. Phys. Rev. Lett. 2019, 123 (4), 048002.

(8) Caraglio, M.; Micheletti, C.; Orlandini, E. Mechanical Pulling of Linked Ring Polymers: Elastic Response and Link Localisation. Polymers 2017, 9 (12), 327.

(9) Amici, G.; Caraglio, M.; Orlandini, E.; Micheletti, C. Topologically Linked Chains in Confinement. ACS Macro Lett. 2019, 8 (4), 442-446.

(10) Payamyar, P.; King, B. T.; Öttinger, H. C.; Schlüter, A. D. TwoDimensional Polymers: Concepts and Perspectives. Chem. Commun. 2016, 52 (1), 18-34.

(11) Colson, J. W.; Dichtel, W. R. Rationally Synthesized TwoDimensional Polymers. Nat. Chem. 2013, 5 (6), 453-465.

(12) Niu, Z.; Gibson, H. W. Polycatenanes. Chem. Rev. 2009, 109 (11), 6024-6046.

(13) Rauscher, P. M.; Rowan, S. J.; de Pablo, J. J. Topological Effects in Isolated Poly[n]catenanes: Molecular Dynamics Simulations and Rouse Mode Analysis. ACS Macro Lett. 2018, 7, 938-943.

(14) Krajina, B.A.; Zhu, A.; Heilshorn, S.C.; Spakowitz, A.J. Active DNA Olympic Hydrogels Driven by Topoisomerase Activity. Phys. Rev. Lett. 2018, 121 (14), na.

(15) Mai, D.J.; Schroeder, C.M. Single Polymer Dynamics of Topologically Complex DNA. Curr. Opin. Colloid Interface Sci. 2016, 26,28

(16) Perkins, T.T.; Smith, D.E.; Chu, S. Single Polymer Dynamics in an Elongational Flow. Science 1997, 276 (5321), 2016-2021.

(17) Smith, D. E.; Chu, S. Response of Flexible Polymers to a Sudden Elongational Flow. Science 1998, 281 (5381), 1335-1340.
(18) Schroeder, C. M.; Babcock, H. P.; Shaqfeh, E. S. G.; Chu, S. Observation of Polymer Conformation Hysteresis in Extensional Flow. Science 2003, 301 (5639), 1515-1519.

(19) Laib, S.; Robertson, R. M.; Smith, D. E. Preparation and Characterization of a Set of Linear DNA Molecules for Polymer Physics and Rheology Studies. Macromolecules 2006, 39, 4115-4119. (20) Mai, D. J.; Marciel, A. B.; Sing, C. E.; Schroeder, C. M. Topology-Controlled Relaxation Dynamics of Single Branched Polymers. ACS Macro Lett. 2015, 4 (4), 446-452.

(21) Klotz, A. R.; Soh, B. W.; Doyle, P. S. Equilibrium Structure and Deformation Response of 2D Kinetoplast Sheets. Proc. Natl. Acad. Sci. U. S. A. 2020, 117 (1), 121-127.

(22) Shapiro, T. A.; Englund, P. T. The Structure and Replication of Kinetoplast DNA. Annu. Rev. Microbiol. 1995, 49 (1), 117-143.

(23) Englund, P. T. The Replication of Kinetoplast DNA Networks in Crithidia Fasciculata. Cell 1978, 14 (1), 157-168.

(24) Liu, B.; Liu, Y.; Motyka, S. A.; Agbo, E. E. C.; Englund, P. T. Fellowship of the Rings: The Replication of Kinetoplast DNA. Trends Parasitol. 2005, 21 (8), 363-369.

(25) Jensen, R. E.; Englund, P. T. Network News: The Replication of Kinetoplast DNA. Annu. Rev. Microbiol. 2012, 66 (1), 473-491.

(26) Balducci, A. G.; Tang, J.; Doyle, P. S. Electrophoretic Stretching of DNA Molecules in Cross-Slot Nanoslit Channels. Macromolecules 2008, 41 (24), 9914-9918.

(27) Tang, J.; Trahan, D. W.; Doyle, P. S. Coil-Stretch Transition of DNA Molecules in Slit-like Confinement. Macromolecules 2010, 43 (6), 3081-3089.

(28) Soh, B. W.; Narsimhan, V.; Klotz, A. R.; Doyle, P. S. Knots Modify the Coil-Stretch Transition in Linear DNA Polymers. Soft Matter 2018, 14, 1689-1698.

(29) Long, D.; Viovy, J. L.; Ajdari, A. Simultaneous Action of Electric Fields and Nonelectric Forces on a Polyelectrolyte: Motion and Deformation. Phys. Rev. Lett. 1996, 76 (20), 3858-3861.

(30) Lee, S. S.; Yim, Y.; Ahn, K. H.; Lee, S. J. Extensional FlowBased Assessment of Red Blood Cell Deformability Using Hyperbolic Converging Microchannel. Biomed. Microdevices 2009, 11 (5), 10211027.

(31) Bento, D.; Rodrigues, R.; Faustino, V.; Pinho, D.; Fernandes, C.; Pereira, A.; Garcia, V.; Miranda, J.; Lima, R. Deformation of Red Blood Cells, Air Bubbles, and Droplets in Microfluidic Devices: Flow Visualizations and Measurements. Micromachines 2018, 9 (4), 151.

(32) Li, Y.; Hsiao, K. W.; Brockman, C. A.; Yates, D. Y.; RobertsonAnderson, R. M.; Kornfield, J. A.; San Francisco, M. J.; Schroeder, C. M.; McKenna, G. B. When Ends Meet: Circular DNA Stretches Differently in Elongational Flows. Macromolecules 2015, 48 (16), 5997-6001.

(33) Mai, D. J.; Saadat, A.; Khomami, B.; Schroeder, C. M. Stretching Dynamics of Single Comb Polymers in Extensional Flow. Macromolecules 2018, 51 (4), 1507-1517.

(34) Larson, R. G.; Hu, H.; Smith, D. E.; Chu, S. Brownian Dynamics Simulations of a DNA Molecule in an Extensional Flow Field. J. Rheol. 1999, 43 (2), 267-304.

(35) Suma, A.; Di Stefano, M.; Micheletti, C. Electric-Field-Driven Trapping of Polyelectrolytes in Needle-like Backfolded States. Macromolecules 2018, 51 (12), 4462-4470.

(36) Gerashchenko, S.; Steinberg, V. Critical Slowing Down in Polymer Dynamics Near the Coil-Stretch Transition in Elongation Flow. Phys. Rev. E 2008, 78, 040801.

(37) Evans, E. A. Bending Elastic Modulus of Red Blood Cell Membrane Derived From Buckling Instability in Micropipet Aspiration Tests. Biophys. J. 1983, 43 (1), 27-30.

(38) Yaginuma, T.; Oliveira, M. S. N.; Lima, R.; Ishikawa, T.; Yamaguchi, T. Human Red Blood Cell Behavior Under Homogeneous Extensional Flow in a Hyperbolic-Shaped Microchannel. Biomicrofluidics 2013, 7 (5), 054110. 